\title{
Factor XII (Hageman Factor) Deficiency: A Very Rare Coagulation Disorder
}

\author{
Mohammad Arphan Azaad, Qiurong Zhang*, Yongping Li \\ Department of Clinical Medical College, Dali University, Dali, Yunnan, China \\ Email: ${ }^{*}$ zqr688990@163.com, arphanazaad@hotmail.com
}

Received 14 September 2015; accepted 20 October 2015; published 23 October 2015

Copyright (C) 2015 by authors and Scientific Research Publishing Inc.

This work is licensed under the Creative Commons Attribution International License (CC BY).

http://creativecommons.org/licenses/by/4.0/

\section{(c) (i) Open Access}

\begin{abstract}
Background: Factor XII (Hageman Factor) is the initiating factor for the Intrinsic Pathway of Coagulation. Very low levels of Factor XII have been associated with increased levels of activated Partial Thromboplastin Time (aPTT). Association of Factor XII deficiency is more with thromboembolic disorders rather than bleeding tendencies. Aim: To learn more about the relationship of factor XII (Hageman Factor) deficiency and high levels of activated Partial Thromboplastin Time. Case Presentation: The Patient was admitted with complains of recurrent headaches and loin pain. Patient was incidentally found to have prolonged activated Partial Thromboplastin Time. This led to investigations which ultimately provided the evidence of severely low levels of Hageman Factor. Conclusion: Hageman Factor deficiency causes prolonged activated Partial Thromboplastin Time. However, most of the patients are asymptomatic for many years despite Hageman Factor deficiency.
\end{abstract}

\section{Keywords}

Factor XII Deficiency, Prolonged aPTT (Activated Partial Thromboplastin Time)

\section{Introduction}

Factor XII, also called Hageman factor, is an enzyme initiating the coagulation cascade. The main laboratory abnormality, which characterizes patients with factor XII deficiency, is an asymptomatic prolongation in activated Partial Thromboplastin Time (aPTT), whose incidence is relatively low (1/1,000,000 individuals). Factor XIIa plays a double role in the cascade reactions of coagulation. It can activate factor XI, which subsequently activates factor IX (previous intrinsic pathway); however, this is its marginal function. The conversion of plasminogen into plasmin by active factor XII and initiation of fibrinolysis is of greater importance. In the human body, the processes of coagulation and fibrinolysis are constantly in a dynamic equilibrium; therefore, factor XII

"Corresponding author. 
deficiency can potentially increase the risk of thrombosis [1]. Congenital factor XII deficiency is not associated with a clinical bleeding tendency; it can be identified on a routine coagulation test, such as a prolonged activated partial thromboplastin time [2]. Factor XII is synthesized in the liver, FXII deficiency is rare and may present as an autosomal recessive or acquired disorder. It is generally asymptomatic. Previously, many studies suggested an association between factor XII (FXII) deficiency and autoantibodies to FXII and recurrent pregnancy loses [3]. Although majority of patients with factor XII deficiency show prolonged activated partial thromboplastin time (aPTT), haemorrhagic incidents are not usually associated with the hereditary form of this factor. On the contrary, these patients suffer more often from thromboembolism as did Hageman himself [4].

Hageman factor was first discovered in 1955 when a routine preoperative blood sample of the 37-year-old railroad brakeman John Hageman was found to have prolonged clotting time in test tubes, even though he had no hemorrhagic symptoms. Hageman was then examined by Dr. Oscar Ratnoff who found that Mr. Hageman lacked a previously unidentified clotting factor. Dr. Ratnoff later found that the Hageman factor deficiency is an autosomal recessive disorder, when examining several related people which had the deficiency. Paradoxically, pulmonary embolism contributed to Hageman's death after an occupational accident. Since then, case series clinical studies have identified an association of thrombosis and factor XII deficiency, though the pathophysiology of the relationship is unclear. Hepatocytes express blood coagulation factor XII [5].

Factor XII (Hageman Factor) deficiency is a rare detection, usually persistent prolongations of activated Partial Thromboplastin Time (aPTT) suggests of Hemophilia which we have ruled out in this patient. Patient had no history of taking Warfarin or any other anticoagulation drugs, so the diagnosis was challenging.

The patient had no visible evidence of internal or external bleeding which led us to suspect factor XII deficiency which ultimately was the etiology of prolonged activated Partail Thromboplastin Time (aPTT) in this case.

\section{Case Report}

51-year-old female with a history of Multiple Right Calculi, recurrent sinusitis was incidentally found to have excessively high aPTT. Patient has no history of DM, HTN, PTB, Hepatitis, patient had undergone ESWL for renal calculi and reconstructive nasal surgery for recurrent sinusitis, Patient had no allergic history. The Patient had menarche at the age of 13, menstrual period is around every 28 to 30 days, and the patient is unmarried. The patient has no history of spontaneous bleeding. During Hospital stay patient was managed with Vitamin K and other supportive treatment. Since there was no evidence of internal or external bleeding, the medical management was not difficult. On USG examination, multiple right sided ureteric stone was detected, Urine Routine Examination showed excessive hematuria, Complete Blood Count, Renal Function Test, Liver Function Test all were within normal limits. The patient's Blood Group is A Positive.

Documented evidence of Persistent elevation of aPTT at our centre is listed in Table 1.

To find out the cause of excessively raised level of aPTT, investigation to measure the concentration of clotting factor was sent which revealed following parameters as listed in Table 2.

Table 1. aPTT value at Our Centre during patient's inpatient stay.

\begin{tabular}{cc}
\hline aPTT & Date Of Investigations \\
\hline 114.4 seconds & $2015-06-26$ \\
180 seconds & $2015-06-26$ (repeated) \\
146.5 seconds & $2015-06-27$ \\
107.1 seconds & $2015-06-30$ \\
\hline
\end{tabular}

Table 2. Measurement of clotting factor in percentage.

\begin{tabular}{ccc}
\hline Clotting Factor & Percentage Detected & Normal Limits \\
\hline VIII & $85.9 \%$ & $50 \%-150 \%$ \\
IX & $46.4 \%$ & $65 \%-150 \%$ \\
XI & $69.4 \%$ & $65 \%-150 \%$ \\
XII & $0.10 \%$ & $50 \%-150 \%$ \\
\hline
\end{tabular}


We could see the severe depletion of Factor XII in this case, which was the first scenario at our centre. So we would like to report this case. Since patient showed no evidence of bleeding during her hospital stay so Vitamin $\mathrm{K}$ was stopped and no any further drugs were added. Patient has been advised for regular follow up at our centre (at least on every 3 month interval) and future possibility of occurrence of symptoms of Coronary Artery Disease and Peripheral Artery Disease has been explained to the patient.

\section{Discussion}

There have been lots of reports of association of Hageman Factor deficiency of Myocardial Infarction, Recurrent Pregnancy loss, so on detection of Hageman Factor Deficiency we clinician must assume the risk of Myocardial Infarction, Recurrent Pregnancy losses are in higher range rather than internal or visible bleeding. Patient those who are detected to have low Hageman Factor have ultimately died of Acute Myocardial Infarction, Pulmonary Embolism. So better risk management is must for the better prognosis of individuals affected with Factor XII deficiency.

Proposed mechanism for activation for Factor XII is listed in Figure 1.

Mechanisms for FXII activation. There are two pathways for FXII (Hageman factor) activation:

Autoactivation upon exposure to negatively charged surfaces and proteolytic activation on cell membranes. FXII autoactivates $(\mathrm{Km}=2.4 \mu \mathrm{M})$ on an artificial or biologic surface such as kaolin or a thrombus to activate FXII to $\alpha$-FXIIa. $\alpha$-FXIIa then activates FXI to FXIa to initiate hemostasis and activates PK to form plasma kallikrein (KAL). KAL cleaves HK to liberate bradykinin, which induces vasodilatation and vascular permeability. KAL also activates the complement system by directly activating complement components C3 and C5 and cleaving $\alpha$-FXIIa to form $\beta$-FXIIa (a soluble light chain enzymatic form [Hageman factor fragment]), which then activates the macromolecular C1qr,s complex to enzymatically active C1r and C1s. The results of the study by Maas et al. in this issue of the JCI (10) suggest that a second FXII autoactivation mechanism occurs upon exposure of FXII to aggregates of misfolded proteins and that this activation results in PK activation without FXI activation—showing that the kallikrein-kinin system can be activated separately from the coagulation cascade by FXII. A second pathway for FXII activation occurs on endothelial cells. PK bound to HK on endothelial cells is activated to plasma KAL by the serine protease prolylcarboxypeptidase (PRCP) $(K \mathrm{~m}=9 \mathrm{nM})$. KAL then activates FXII to $\alpha$-FXIIa $(K \mathrm{Km}=11 \mu \mathrm{M})$. FXII

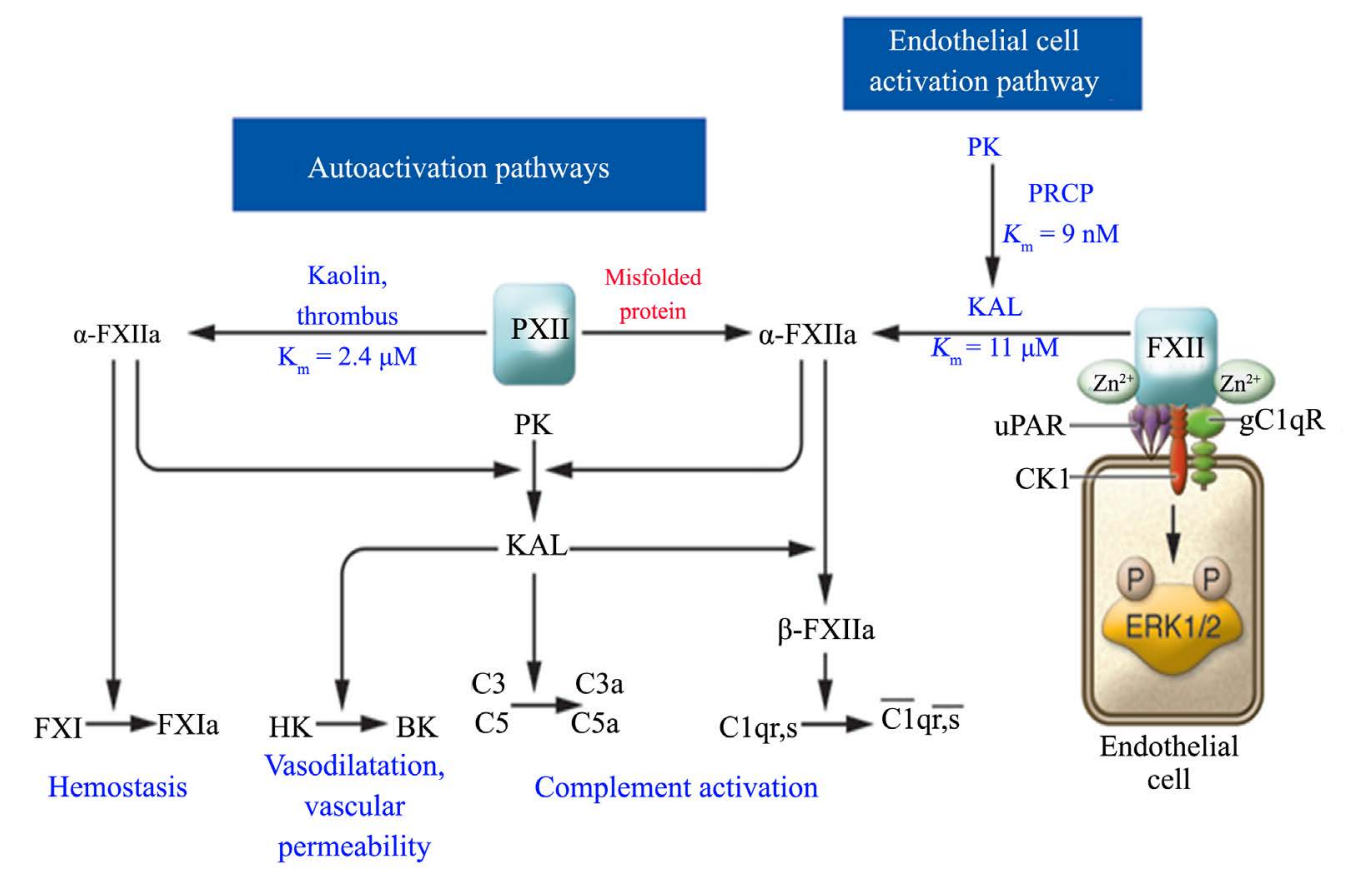

Figure 1. Mechanism for Factor XII activation [6]. 
also binds to endothelial cells in the presence of zinc ions and when bound stimulates ERK1/2 phosphorylation. There is explanation of the phosphorylation and subsequent activation of Urokinase Plasminogen activator receptor.

It is a challenge for further management of case who is found to have low Factor XII, because although aPTT is prolonged but no obvious bleeding symptoms are manifested.

In recent years, the fibrinolysis effect of coagulation factor XII attracts more and more concerns and is thought to be greater than the effect of coagulation factor XII in intrinsic coagulation pathway. Currently, researches on coagulation factor XII are mainly focused on cardiovascular and cerebrovascular diseases [7].

Coagulation factor XII deficiency is either congenital or acquired. Congenital coagulation factor XII deficiency is a kind of rare autosomal recessive hereditary disease, and its clinical manifestation is low factor concentration without significant bleeding. Therefore, coagulation factor XII deficiency is often diagnosed before surgeries or during routing coagulation tests. Because no severe bleeding is related to this disease, no special treatment is required. Acquired coagulation factor XII deficiency is extremely rare and is related to coagulation factor XII inhibitors produced in patients with leukemia or nephrotic syndrome [7].

There are several conditions resulting in an isolated prolonged APTT. Deficiencies in contact factors, factors VIII, IX, XI and XII deficiencies, all result in an isolated APTT prolongation, as well as lupus anticoagulant, anticardiolipin, Von Willebrand disease and acquired clotting factor inhibitors such as acquired haemophilia A [8]. This lack of a bleeding tendency observed with FXII deficiency is in sharp contrast to deficiencies of other components of the coagulation cascade, such as FVII, tissue factor (TF) and FVIII or FIX (causing the bleeding disorders hemophilia A and B, respectively) and has led to the reasonable hypothesis that fibrin formation in vivo is initiated largely, if not exclusively, through the extrinsic pathway of coagulation [9].

\section{Conclusions}

$\checkmark$ Since most of the patients detected with factor XII deficiency are asymptomatic, so no immediate modes of treatment will be required.

$\checkmark$ Rather than bleeding tendency, Hageman Factor deficiency is associated with possible thrombotic occlusive disorders.

$\checkmark$ Factor XII (Hageman Factor) is a rare but important cause of prolongation of activated Partial Thromboplastin Time (aPTT).

\section{References}

[1] Rygal, P. and Kuc, A. (2012) Perioperative Management of Cardiac Surgery Patients with Factor XII Deficiency-Two Case Reports. Anesthesiology Intensive Therapy, 44, 217-220.

[2] Mariano, M.L.,Yadira, P., Ana, A., Manuel, L. and Lopez Galvez, J.J. (2011) Coagulation Factor XII Congenital Deficiency in Women with Recurrent Miscarriage. International Journal of Clinical Medicine, 2, 469-472

[3] Ozgu-Erdinc, A.S., Togrul, C., Aktulay, A., Buyukkagnici, U., Yapar Eyi, E.G. and Erkaya, S. (2014) Factor XII (Hageman) Levels in Women with Recurrent Pregnancy Loss. Journal of Pregnancy, 2014, Article ID: 459192

[4] Ganguli, P., Rigvardhan and Kotwal, J. (2015) Thrombosis: Presentation of a Factor XII Deficiency in 10 Months Old Child-A Rare Case. International Journal of Advances in Case Reports, 2, 708-710.

[5] Ratnoff, O.D. and Margolius Jr., A. (1955) Hageman Trait: An Asymptomatic Disorder of Blood Coagulation. Transactions of the Association of American Physicians, 68, 149-54.

[6] Schmaier, A.H. (2008) The Elusive Physiologic Role of Factor XII. Journal of Clinical Investigation, 118, 3006-3009. http://dx.doi.org/10.1172/jci36617

[7] Wang, Y., Wang, Z., Li, H., Bi, K. and Zhu, C. (2014) Essential Thrombocytosis Accompanied by Coagulation Factor XII Deficiency: A Case Report. International Journal of Clinical and Experimental Medicine, 7, 3725-3729.

[8] Nizamoglu, M., Alexander, K.S., Anwar, U. and Bhandari, S. (2014) Isolated APTT Prolongation-Not Always a Bleeding Risk in Acute Paediatric Burns Surgery. European Journal of Plastic Surgery, 37, 695-696. http://dx.doi.org/10.1007/s00238-014-1012-y

[9] Renné, T., Schmaier, A.H., Nickel, K.F., Blombäck, M. and Maas, C. (2012) In Vivo Roles of Factor XII. Blood, 120, 4296-4303. http://dx.doi.org/10.1182/blood-2012-07-292094 